\title{
Dispersion and Orbit Distortions in the Tevatron: A Comparison of Measurements with a Computer Model.
}

$\underline{\text { Norman Gelfand }}$

Fermilab

Beams Division

Beam Physics

$\underline{\text { May 13, } 2003}$

Acknowledgement

Operated by Universities Research Association Inc under Contract No. DE-AC02-76CH03000 with the United States Department of Energy. 


\section{Introduction.}

In order to improve the Tevatron's performance an accurate model would be of great value. To create a realistic model of the current accelerator requires that we be able to integrate information from measurements made on the Tevatron into the computer model. The data, obtained from experiments on the Tevatron, can constrain the model and also constitute a test of the model. This note will describe comparisons of a computer model of the Tevatron with measurements. All the comparisons are for the injection lattice at $150 \mathrm{GeV}$.

\section{Description of the Computer Model of the Tevatron.}

The description of the base lattice used in the calculations comes from the MAD file found on the Fermilab web site. (The URL for the lattice is http://www-ap.fnal.gov/ martens/mad/tevatron/tevgen2.lat.) This file has been converted into a format suitable for use with Tevlat. Tevlat is a tracking code originally developed by A. Russell ${ }^{1}$.

The Tevatron lattice contains, in addition to the focusing quadrupoles and bend magnets, many additional kinds of elements. Among these are dipole correctors and non-linear elements, such as the chromaticity sextupoles. In addition the lattice quads and dipoles have non-linear fields described with high order multipoles (HOM). In Tevlat these non-linearities are handled as zero length kicks, that is as changes in the slope of the particle's trajectory as it goes though a non-linear element. As a result Tevlat is a symplectic code.

The HOM in the Tevatron magnets were measured as the magnets were constructed in the early part of the 1980's. These measurements are included in the calculations discussed below. Recent measurements made on the settings of the smart bolts on the dipole magnets indicate that there has been relative movement of the coils in the magnets. These measurements suggest that the $a_{l}$ coefficient in the expansion of the magnet's field has increased by approximately 1.4 units $^{2}$ since the magnets were measured. These new measurements were critical to our ability to construct a realistic model for the Tevatron.

The sextupole component of the magnetic field in the dipole magnets is not uniform along the length of the magnet. The sextupole component at the ends is compensated by the sextupole moment of the body of the magnet to yield a small net sextupole for the dipoles. This characteristic of the magnets is incorporated into the model.

Additional data that are incorporated into the model are the measurements of the rolls of both the dipoles and quadrupole magnets. The rolled quads contribute to the coupling in the Tevatron. The rolled dipoles create a closed orbit distortion, primarily in the vertical plane. This closed orbit distortion can be corrected using the vertical dipole correctors located in the Tevatron and included in the model.

The values of the correction elements in the Tevatron lattice, the tuning quadrupoles, the chromaticity sextupoles, the skew quadrupoles were adjusted in the model to give the following values for the tune and chromaticity:

\section{Table I Tunes and Chromaticity}

\footnotetext{
${ }^{1}$ A. Russell, private communication.

${ }^{2}$ The magnetic field at a point $(x, y)$ in the magnet is given by $B_{y}+i B_{x}=B_{0} * \sum_{n}\left(b_{n}+i a_{n}\right) *(x+i y)^{n}$. $B_{0}$ is the nominal dipole field of the magnet. At Fermilab the coefficients $b_{n}$ and $a_{n}$ are quoted as in units which are $10^{4} \bullet\left(b_{n}, a_{n}\right)$ when $(x, y)$ are measured in inches.
} 


\begin{tabular}{|c|c|c|}
\hline & $v$ & $\zeta$ \\
\hline $\mathrm{x}$ & 20.5826 & 8.400 \\
\hline $\mathrm{y}$ & 20.5755 & 8.200 \\
\hline
\end{tabular}

The values of the correction elements found by the program are compared with the values used in the Tevatron in Table II

Table II

Comparison of the Strengths of the Correction Elements

Tevlat Calculation vs. Tevatron Settings $(150 \mathrm{GeV} / \mathrm{c})$

\begin{tabular}{|c|c|c|c|c|}
\hline $\begin{array}{l}\text { Correction } \\
\text { Circuits } \\
\end{array}$ & $\begin{array}{l}\text { Tevlat } \\
\text { Calculation }\end{array}$ & $\begin{array}{l}\text { Tevatron } \\
\text { Setting } \\
\end{array}$ & $\underline{\text { Diff }}$ & $\underline{\text { Ratio }}$ \\
\hline Tuning quad-1 & $1.2106 \mathrm{E}-02$ & $1.2973 \mathrm{E}-02$ & $-8.6742 \mathrm{E}-04$ & 0.93 \\
\hline Tuning quad-2 & $-2.8143 \mathrm{E}-02$ & $-3.0020 \mathrm{E}-02$ & $1.8770 \mathrm{E}-03$ & 0.94 \\
\hline Chro. Sext. F & $6.1225 \mathrm{E}-02$ & $2.7376 \mathrm{E}-02$ & $3.3849 \mathrm{E}-02$ & 2.24 \\
\hline Chro. Sext. D & 4.9684E-03 & $6.9946 \mathrm{E}-03$ & $-2.0262 \mathrm{E}-03$ & 0.71 \\
\hline Skew quad. ksq & 8.3797E-04 & $8.7013 \mathrm{E}-04$ & $-3.2157 \mathrm{E}-05$ & 0.96 \\
\hline Skew quad. ksqa0 & $-1.0674 \mathrm{E}-03$ & -1.8919E-03 & 8.2456E-04 & 0.56 \\
\hline Skew quad. ksqa4 & 1.4054E-03 & $1.5582 \mathrm{E}-03$ & $-1.5282 \mathrm{E}-04$ & 0.90 \\
\hline Skew quad. ksqb1 & $-3.2192 \mathrm{E}-04$ & $-1.6915 \mathrm{E}-04$ & $-1.5276 \mathrm{E}-04$ & 1.90 \\
\hline
\end{tabular}

The agreement between the strengths of the correctors as computed by Tevlat and the values used in the Tevatron is generally good. The difference in the strengths of the chromaticity sextupoles is probably due to poor knowledge of the sextupole multipole, $b_{2}$, in the dipoles. The values of the $b_{2}$ in the dipoles in the early part of the cycle, are changing rapidly due to the "snap back" as well as the acceleration ramp.

\section{Comparison of Measurements With the Model}

\section{The Strengths of the Vertical Dipole Correctors}

As mentioned above the measured rolls were incorporated into the model of the Tevatron. The closed orbit was corrected in Tevlat using the vertical and horizontal dipole correctors (DFG). A comparison of the values of the strengths of the vertical DFG actually used in the Tevatron with those calculated with Tevlat to "smooth" the closed orbit will test both the model and the reliability of the measured rolls. Figure 1 shows the comparison. ${ }^{3}$

\footnotetext{
${ }^{3}$ In all the figures that follow where we have both Tevlat calculations and measurements, the calculations will be the solid line and the measurements the dotted line with the X.
} 


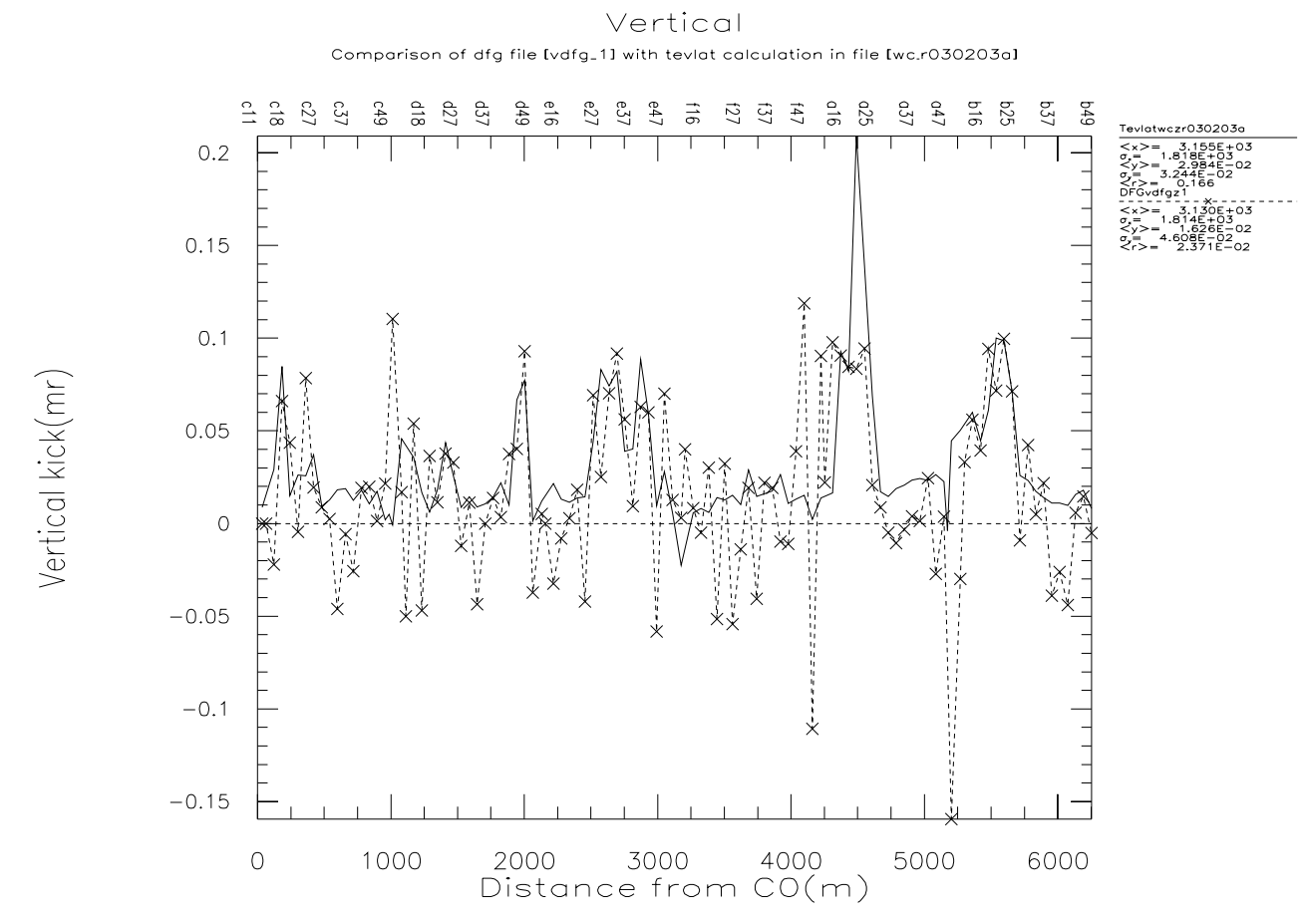

Figure 1. Comparison of Calculated Strengths of the Vertical DFG With Tevatron Settings.

The agreement is generally excellent except near A18. I suspect that the operators in the control room, rather than have a large kick in a single vertical DFG, put the required kick into two adjacent vertical DFGs. Tevlat has not been programmed to do that. The deviations around $\mathrm{B} 0$ and $\mathrm{A} 0$ are probably due to obit bumps not programmed into Tevlat.

Using the measured rolls of the dipoles the calculated strengths of the vertical DFG agrees well with the values used to correct the closed orbit in the Tevatron. I therefore conclude that the values of vertical DFG are determined, primarily, by the rolls of the dipoles and that the rolls are well measured in the survey. Thus correcting the rolls should reduce the settings in the vertical DFG.

\section{The Dispersion}

In the absence of coupling there would be no vertical dispersion in the Tevatron. Thus a non-zero measurement for the vertical dispersion indicates coupling. The sources of any coupling include the rolls in the lattice quadrupoles, skew sextupole correctors used in the feed down circuits, and the skew moments $\left(a_{n}\right)$ in the dipoles and quadrupoles in the lattice. The primary source of the coupling seems to be the $a_{l}$ in the dipoles.

Valeri Lebedev has made extensive measurements of the dispersion in the Tevatron ${ }^{4}$. The results of the Tevlat calculations are compared with the results of his measurements.

Figure 2 shows a comparison of the horizontal dispersion with the Tevlat calculation using the model described above. The value of $d p / p$ used to compare the measurements with the calculation was obtained by

\footnotetext{
${ }^{4}$ Valeri Lebedev, private communication. The data discussed here is a small fraction of the data he has taken. Only a small portion of his data will be used in the comparison discussed here. A larger sample of the data has been analyzed with results consistent with those discussed here.
} 
normalizing the measured BPM data with the Tevlat calculation of the dispersion. ${ }^{5}$ The agreement between the measurements and the calculation is good. Figure 3 shows the difference in the measured readings of the BPM with the values expected from the calculation. ${ }^{6}$ The difference is consistent with the resolution of the BPM of $140 \mu \cdot \sqrt{2}=0.2 \mathrm{~mm}^{7}$

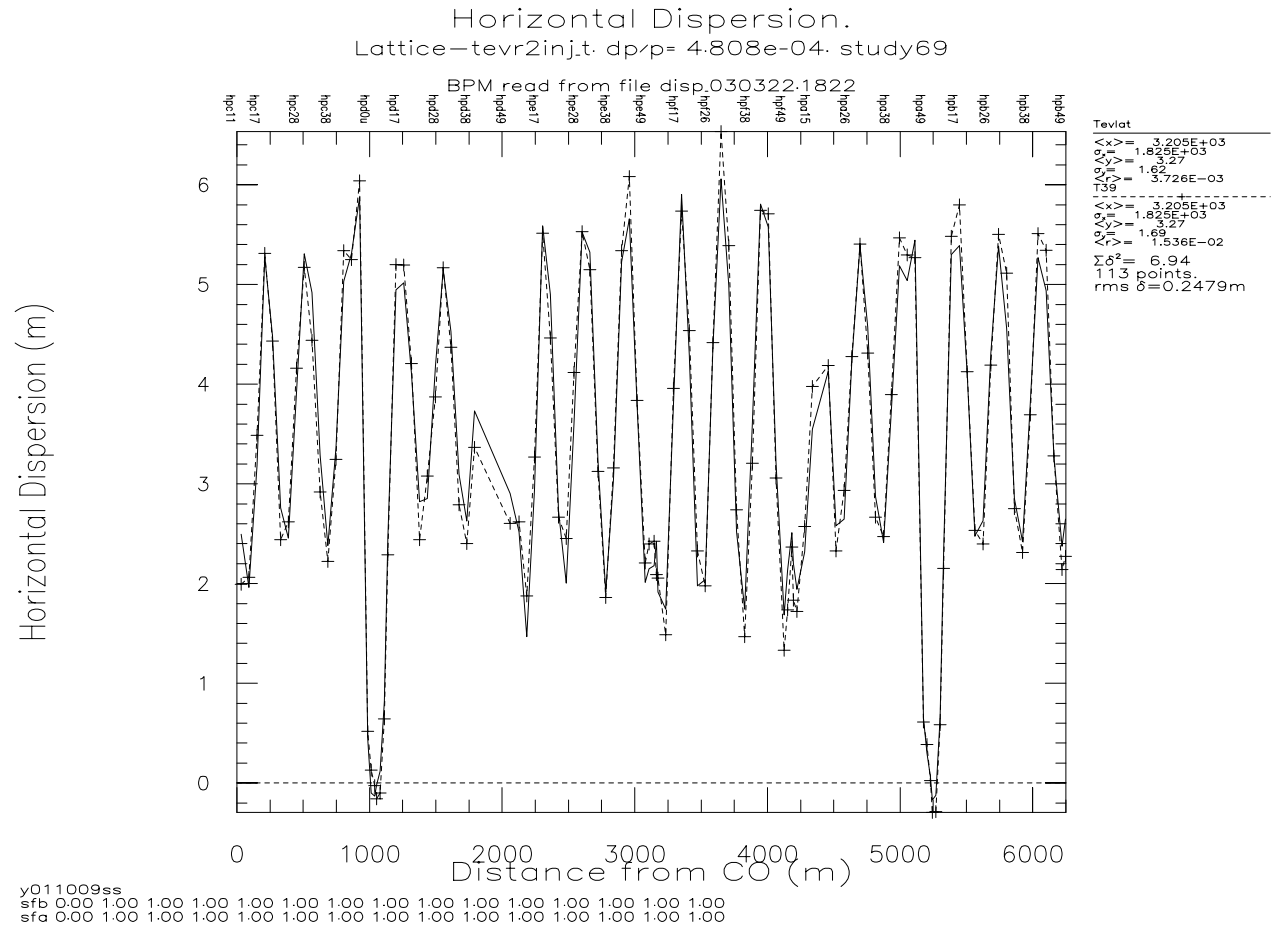

Figure 2 Horizontal Dispersion, Measured and Calculated.

${ }^{5}$ The calculated value of $\delta p / p \sim 4.8 \mathrm{e}-04$ differs from the value of $\delta p / p \sim 5.2 \mathrm{e}-04$ calculated from the change in frequency $\delta f=80 \mathrm{~Hz}$ and the transition gamma $\gamma_{\mathrm{t}}$. The difference corresponds to a change in $\gamma_{\mathrm{t}}$ from the calculated value of $\sim 18.6$ to a value of $\sim 17.8$. Since the transition gamma was calculated from an uncoupled model I regard the agreement as satisfactory.

${ }^{6}$ I show the difference in the BPM values, as I want to compare it to the BPM resolution.

${ }^{7}$ The factor of the $\sqrt{2}$ comes from the fact the BPM readings are the difference between the modified orbit and a reference orbit. 


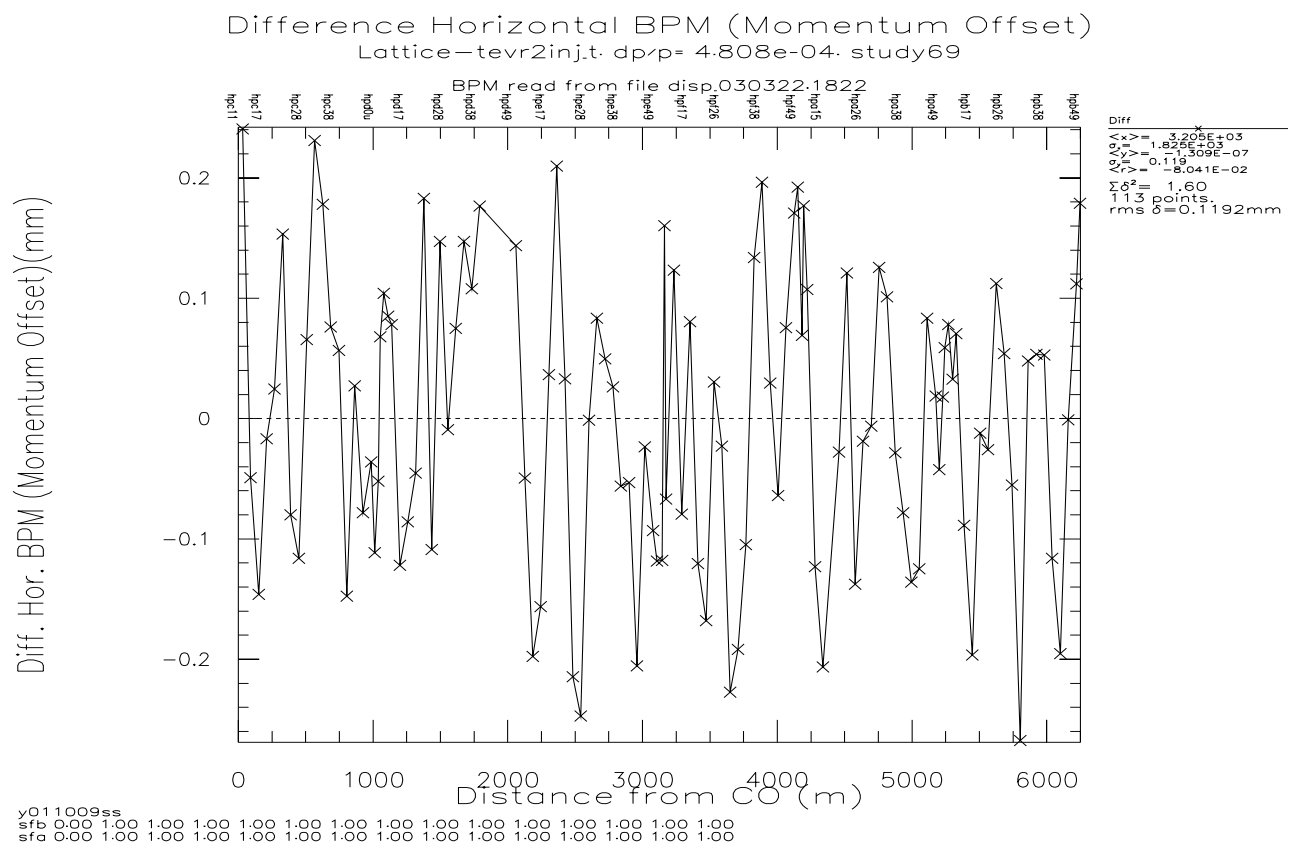

Figure 3 The Difference Between the Measured and Calculated Values of the BPM.

In an uncoupled model of the lattice there would not be any vertical dispersion. The ability to correctly calculate the vertical dispersion is a critical test of our model. The coupling produces the measured vertical dispersion shown in Figure 4 along with the calculation from the model. Figure 5 shows the difference in the measured BPM values and the calculated values. The agreement is quite good, difference being consistent with the uncertainty in the measured values of the BPM.

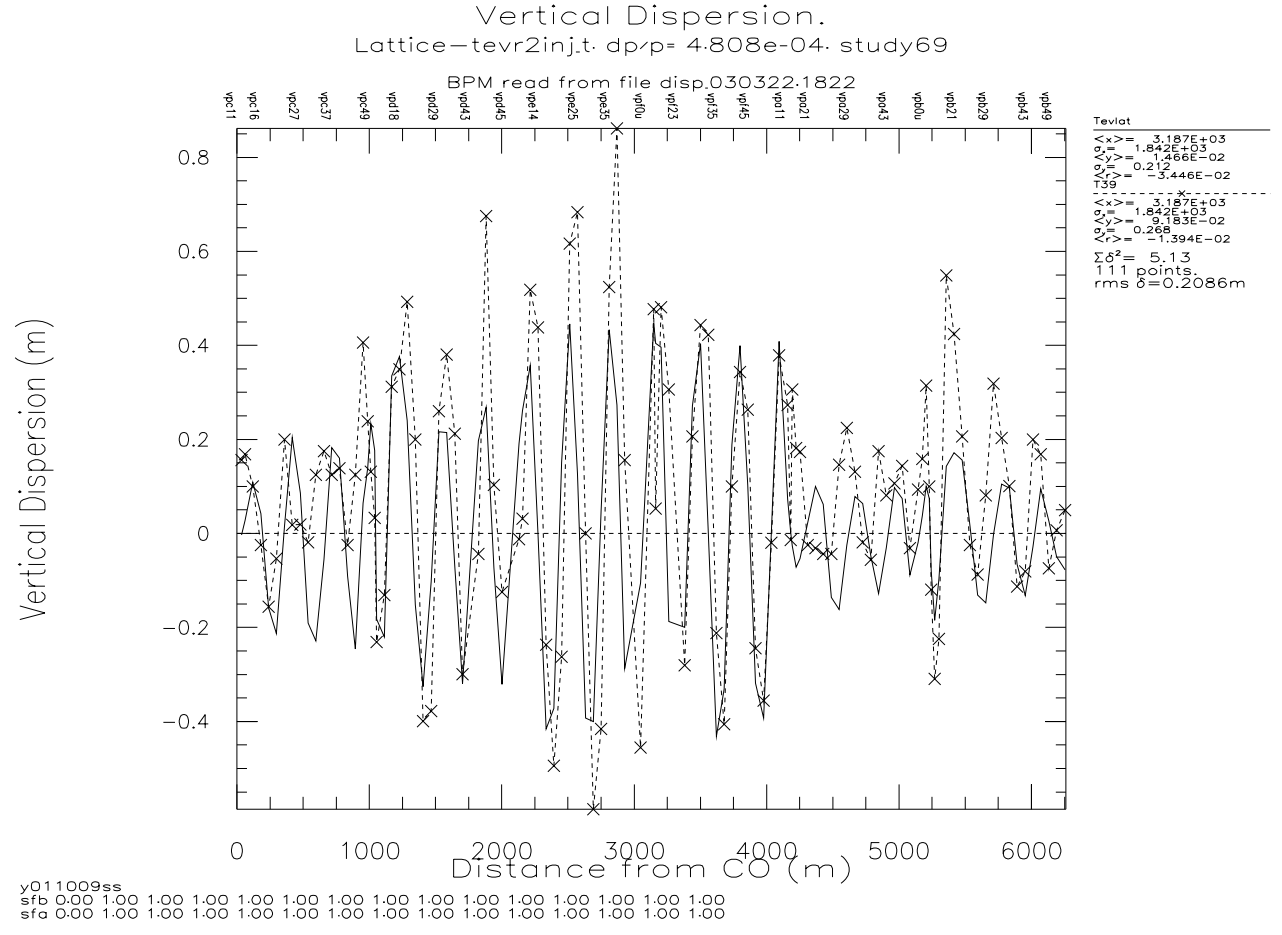

Figure 4 The Vertical Dispersion, Measured and Calculated 


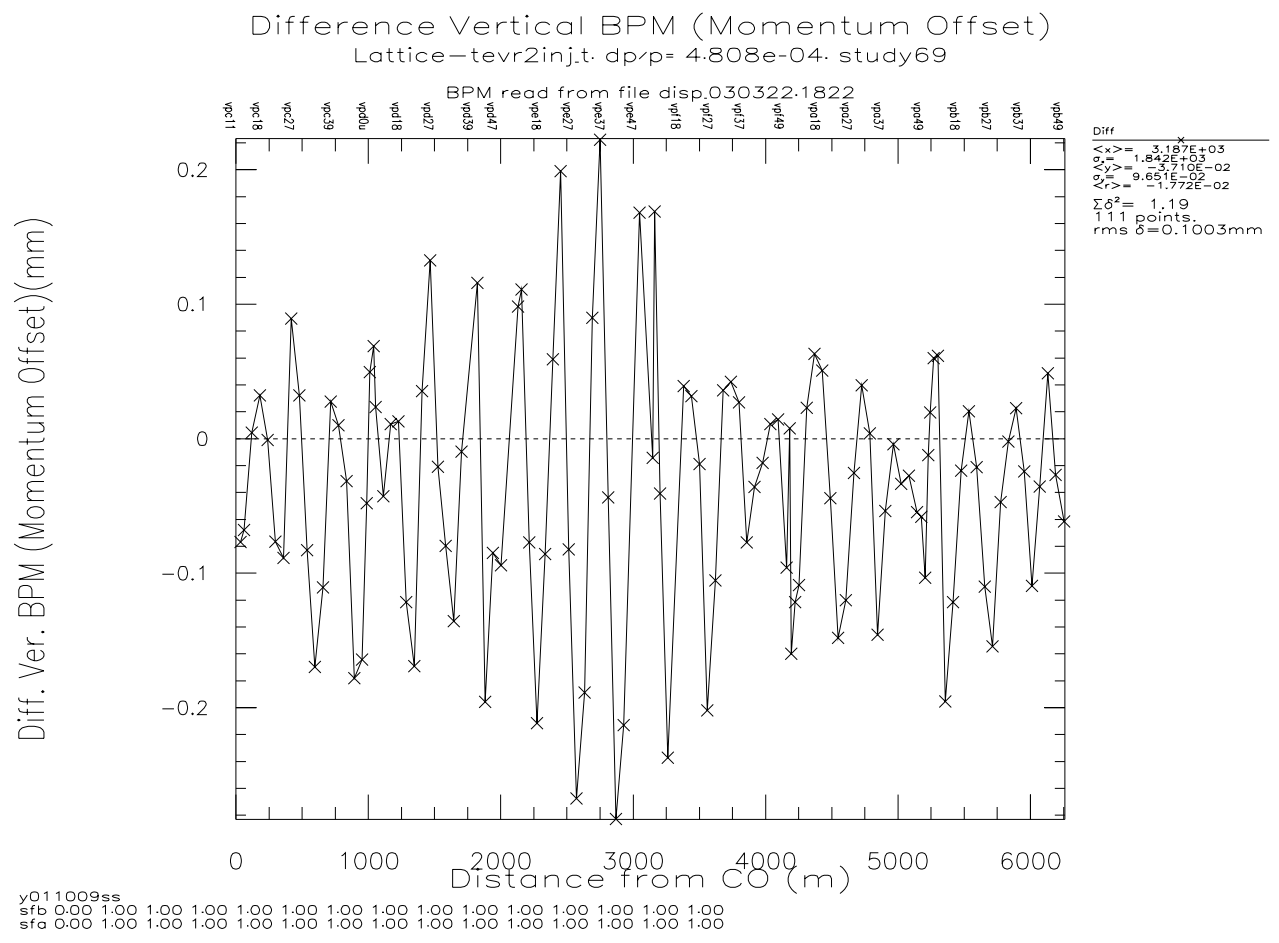

Figure 5 The Difference Between the Measured and Calculated Values of the BPM.

\section{Changes in the Closed Orbit Due to One Bumps}

If the strength of a dipole corrector is changed then there will be a change in the closed orbit. The measured change in the closed orbit due to a known bump can be compared to the change calculated from the model. The change in the orbit in the crossed plane, i.e. the transverse plane perpendicular to the plane of the kick is the significant test of the model since it arises from both linear and non-linear coupling. The following data are representative of the larger data set analyzed.

Figure 6 shows the measured and calculated closed orbit due to a horizontal kick of $\sim 50 \mu r$ located at E42 in the Tevatron. Figure 7 shows the difference between the measured and calculated values. The agreement is quite good; the rms of the differences is $0.2 \mathrm{~mm}$, consistent with our expected resolution. 


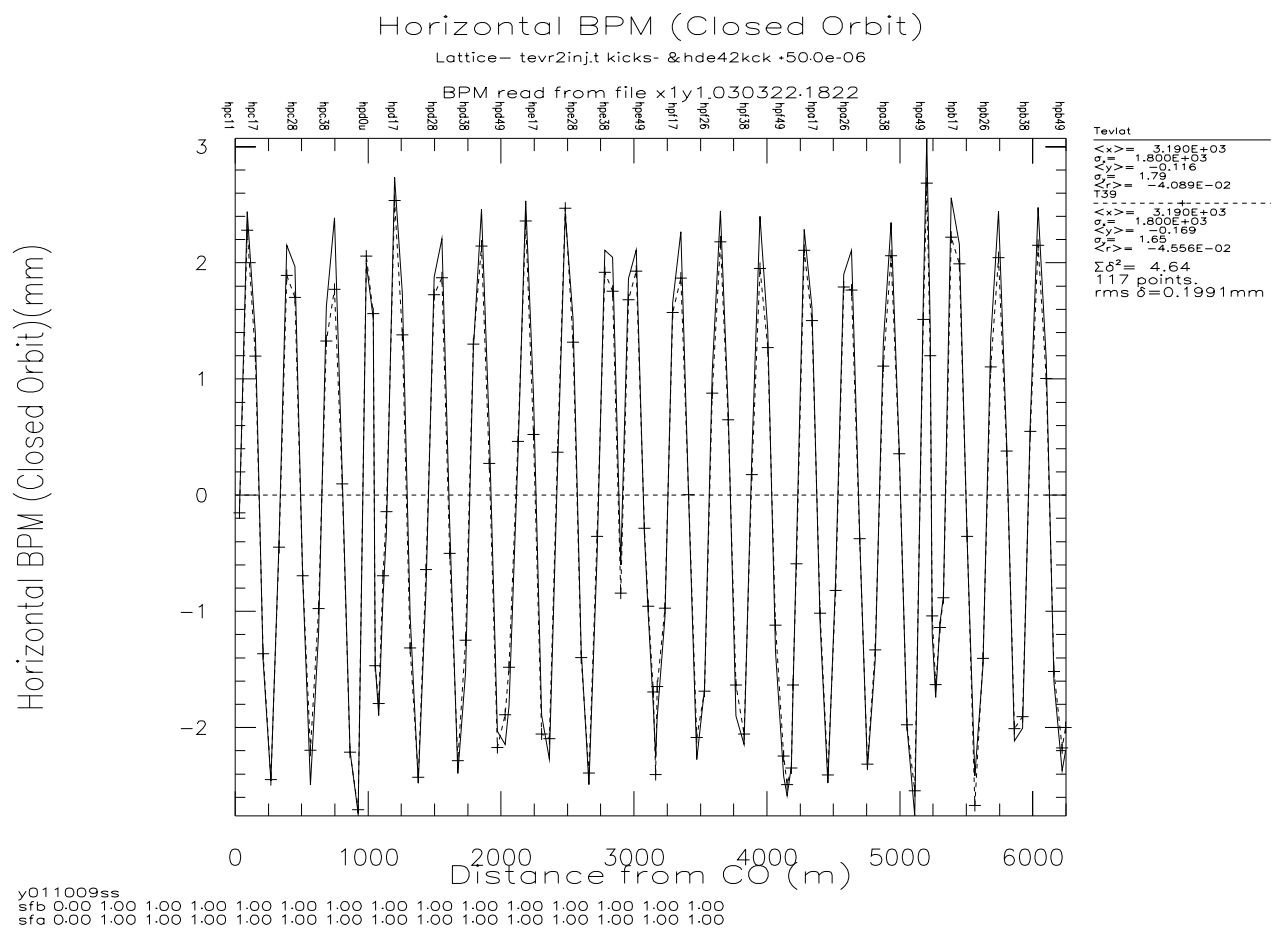

Figure 6 The Measured and Calculated BPM Values of the Horizontal Closed Orbit Resulting from a 50 $\mu r$ Horizontal Kick at E42.

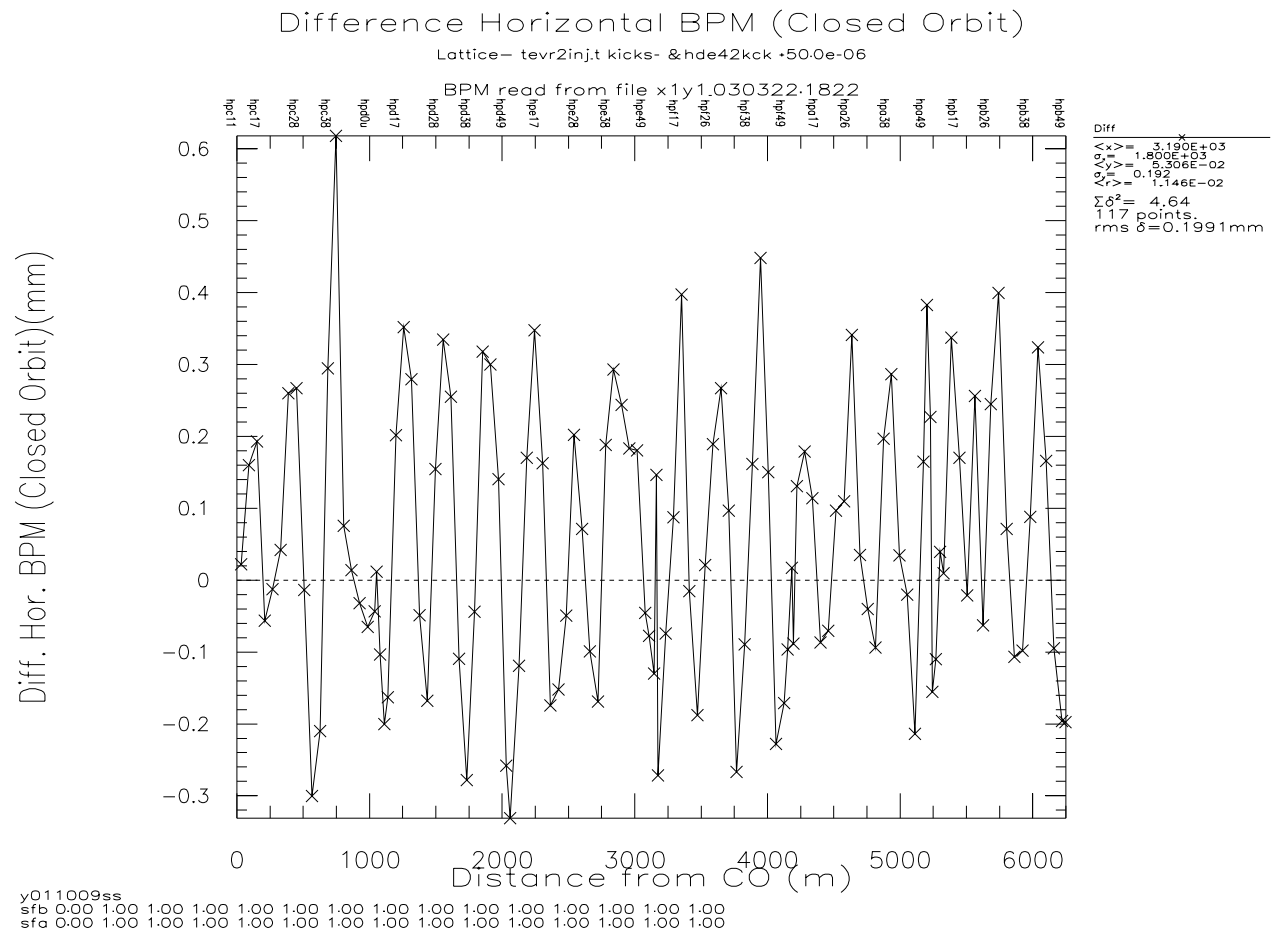

Figure 7 Difference Between the Measured and Calculated Values of the Horizontal BPM Resulting from a 50 $\mu$ r Horizontal Kick at E42. 


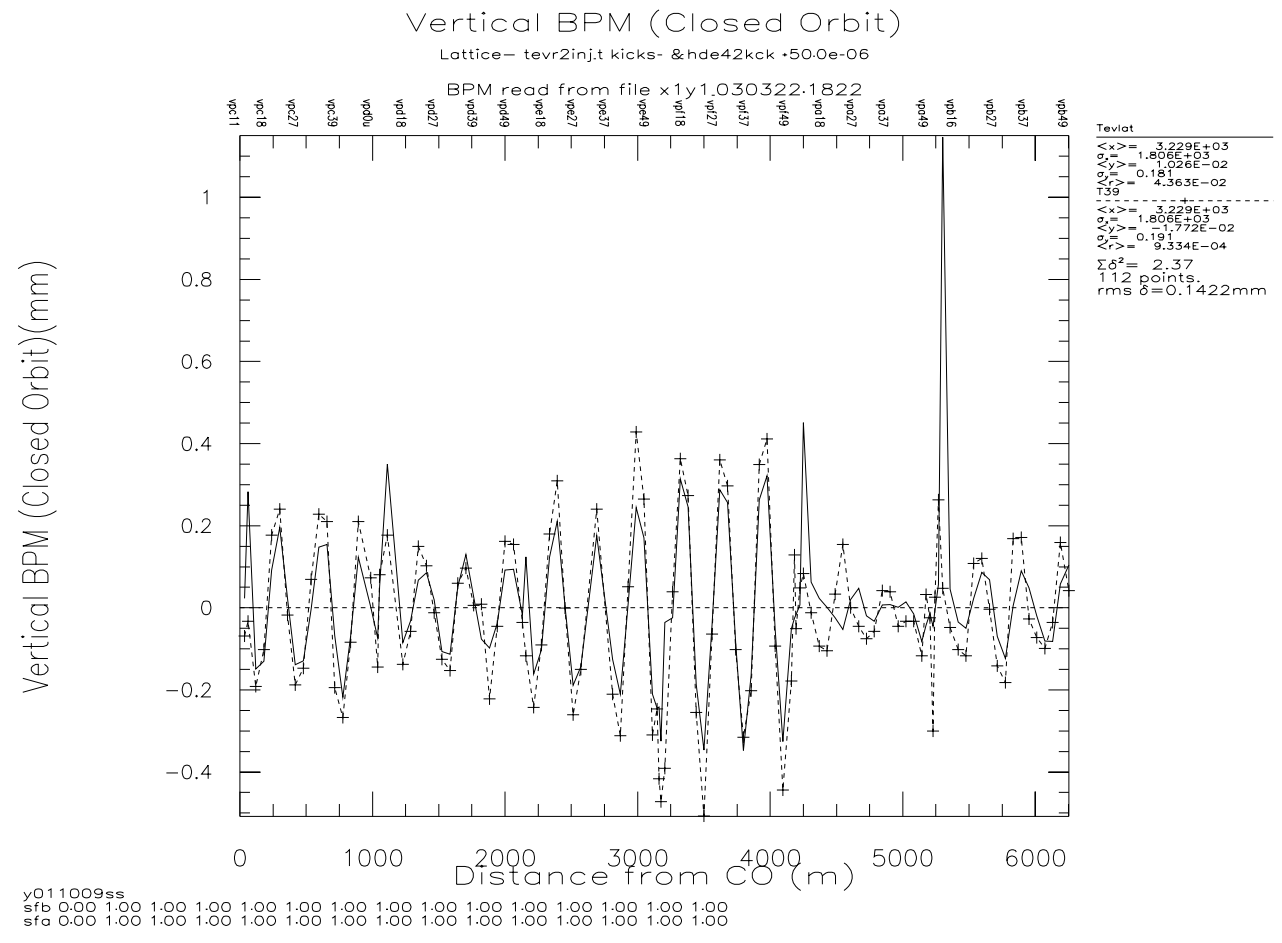

Figure 8 The Measured and Calculated BPM Values of the Vertical Closed Orbit Resulting from a 50mr Horizontal Kick at E42.

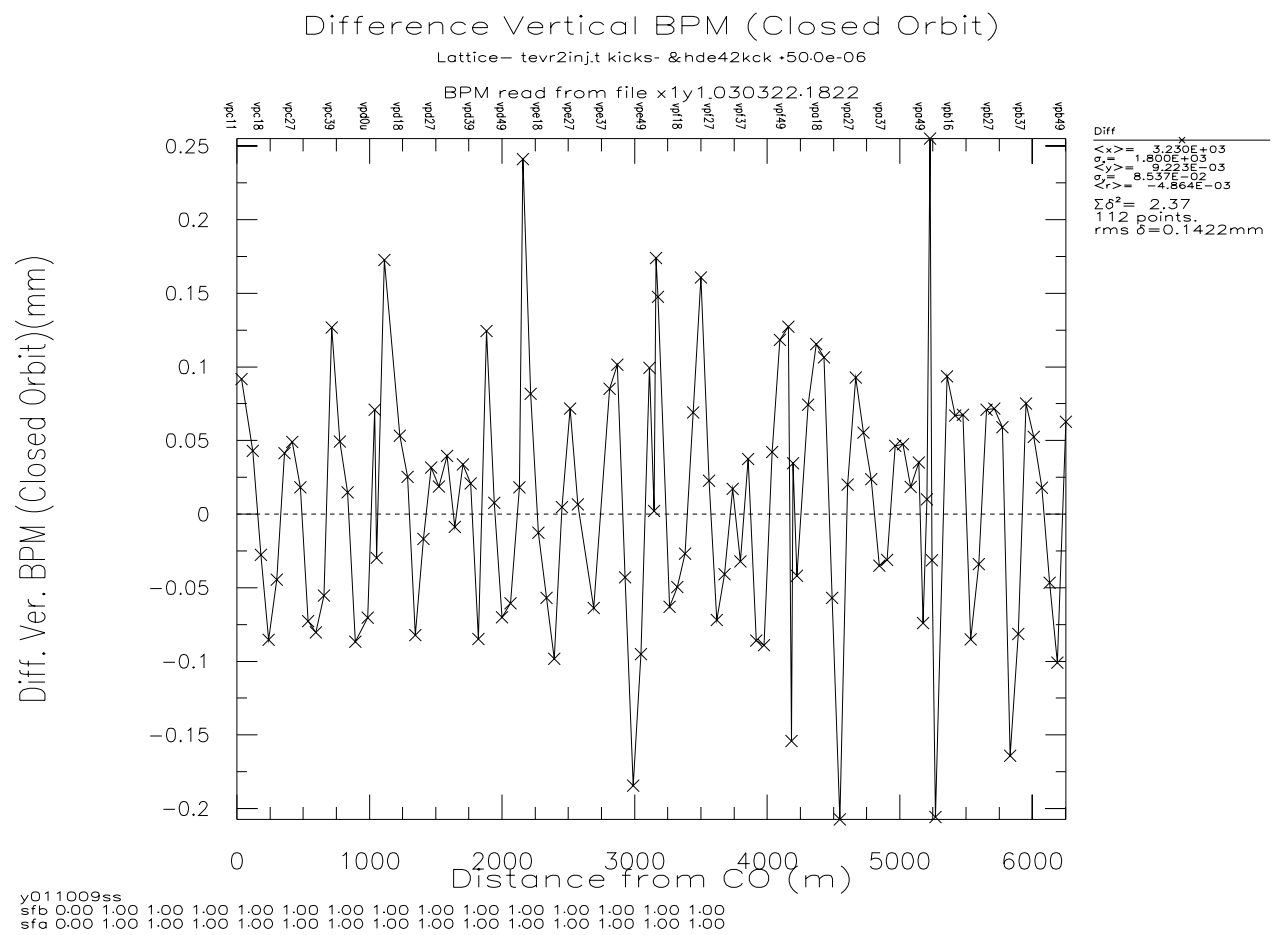

Figure 9 Difference Between the Measured and Calculated Values of the Vertical BPM Resulting from a 50 $\mu$ r Horizontal Kick at E42. 
Figure 8 shows the values of the vertical closed orbit resulting from the same horizontal kick at E42. Figure 9 is the difference between the calculated and measured values. In general the agreement is very good. ${ }^{8}$

We can also study the change in the closed obit when we kick the beam vertically. The measured and calculated values for the closed orbit for a vertical kick of $-50 \mu \mathrm{r}$ are plotted in Figure 10. Figure 11 is a plot of the difference between the measured and calculated values of the closed orbit. Figure 12 is the resulting horizontal closed obit from the vertical kick and Figure 13 is the difference between the calculated and measured values. Again we see good agreement, not only in the plane of the kick but also in the crossed plane.

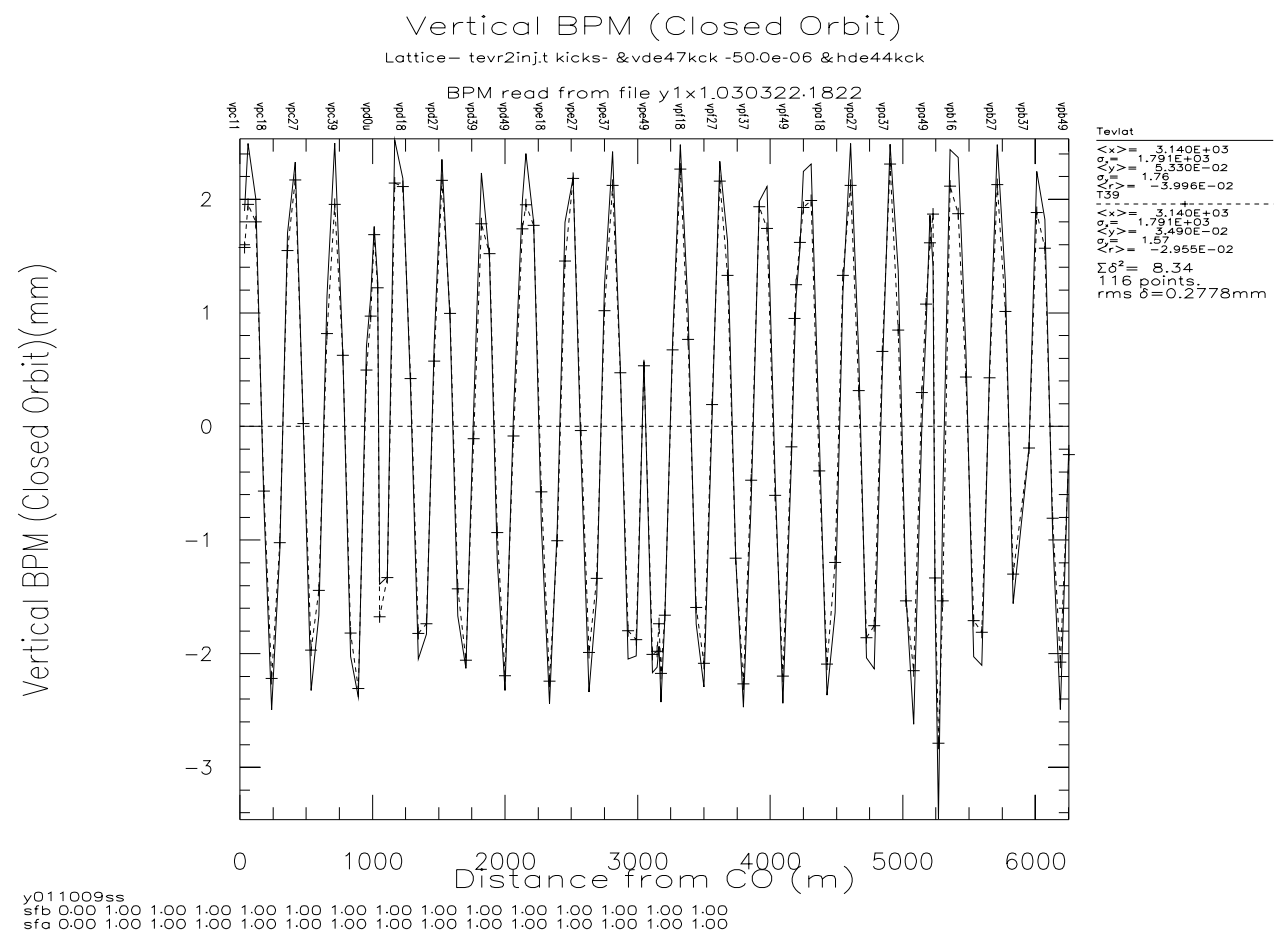

Figure 10 The Measured and Calculated BPM Values of the Vertical Closed Orbit Resulting from a -50 $\mu$ r Vertical Kick at E47.

\footnotetext{
${ }^{8}$ The large spike in the plot of the measured values is almost certainly due to a bad BPM reading. The spike is removed in Figure 9.
} 


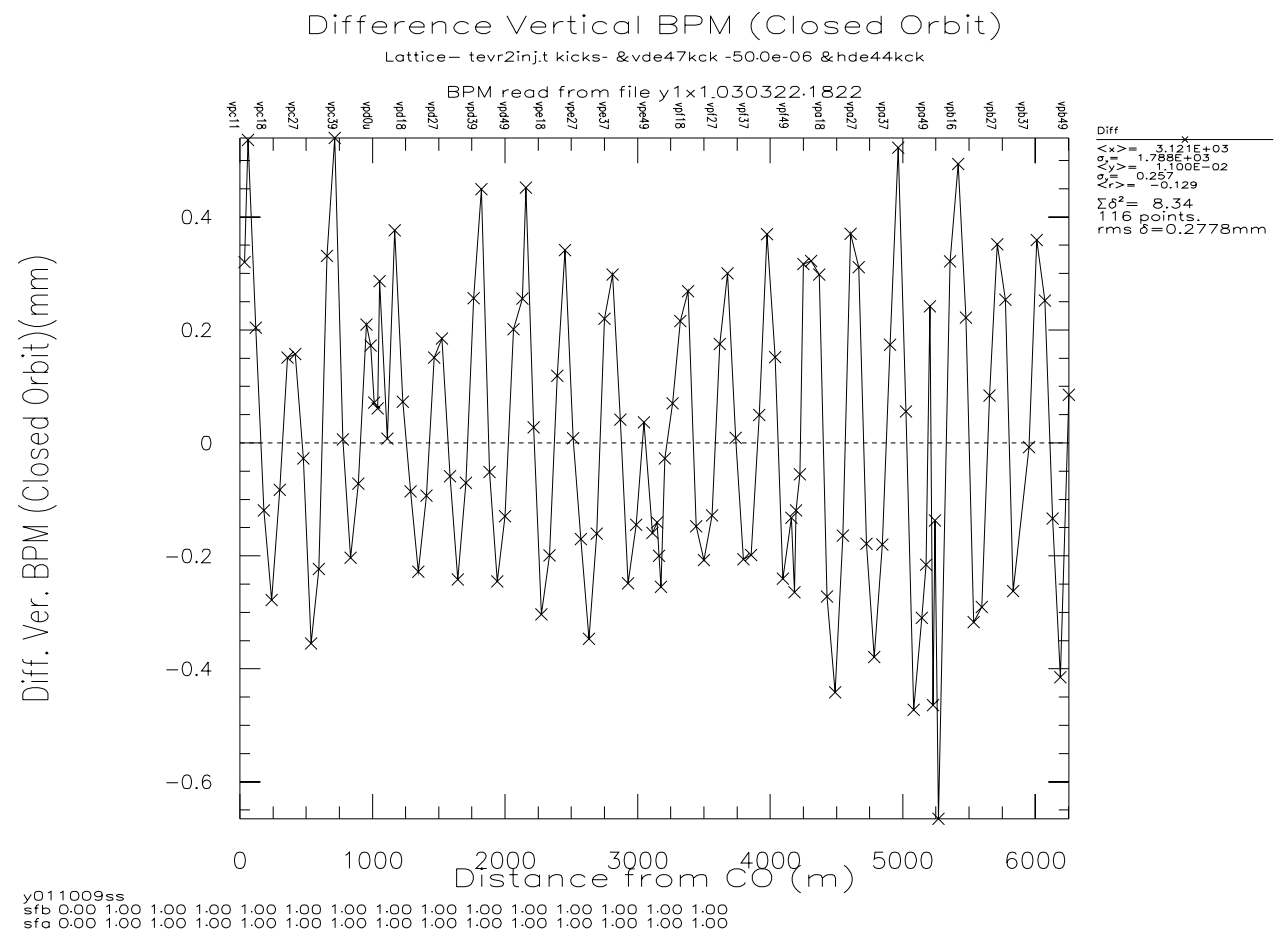

Figure 11 Difference Between the Measured and Calculated Values of the Vertical BPM Resulting from a -50 $\mu$ r Vertical Kick at E47.

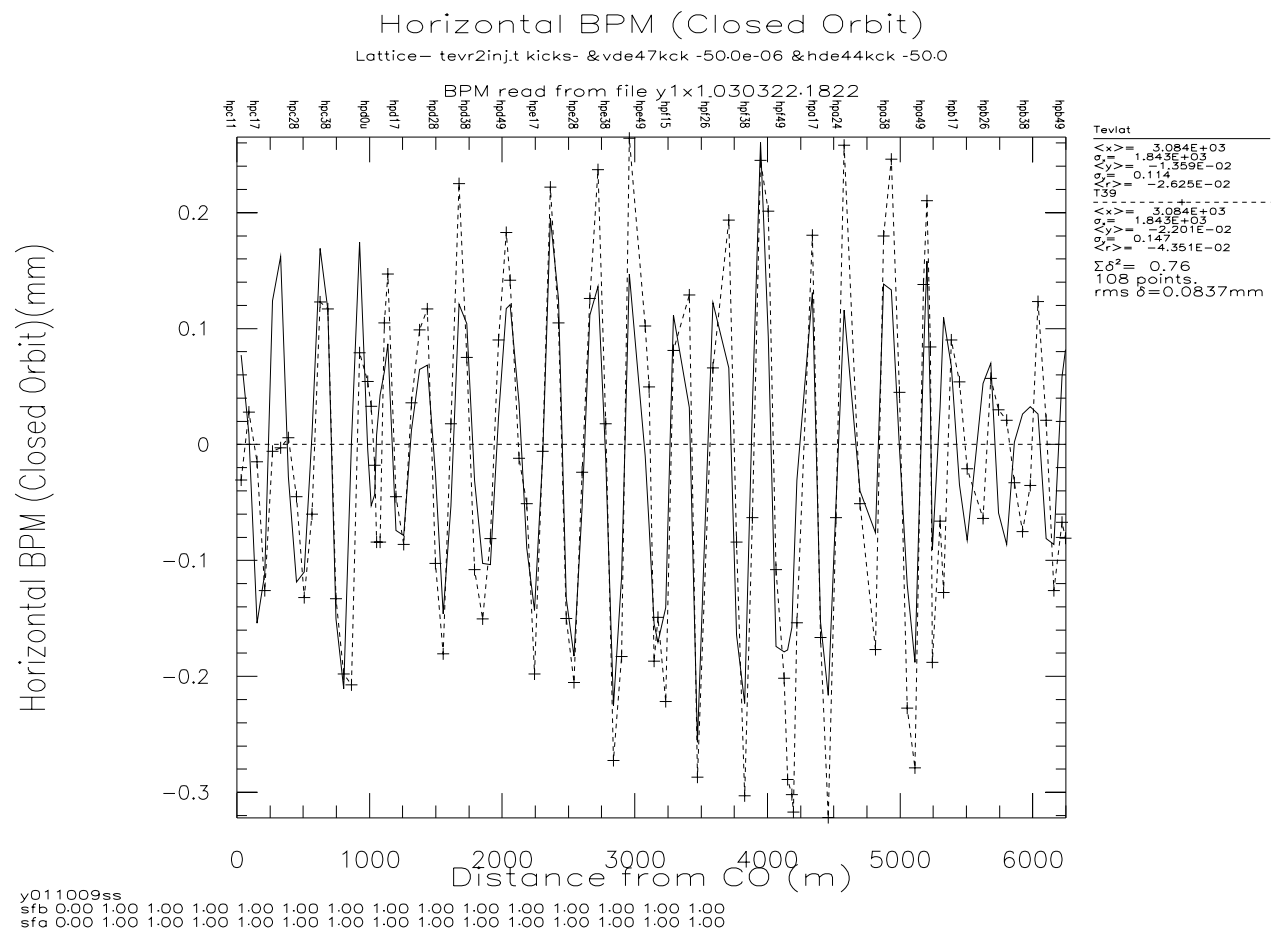

Figure 12 The Measured and Calculated BPM Values of the Horizontal Closed Orbit Resulting from a -50 $\mu$ r Vertical Kick at E47. 


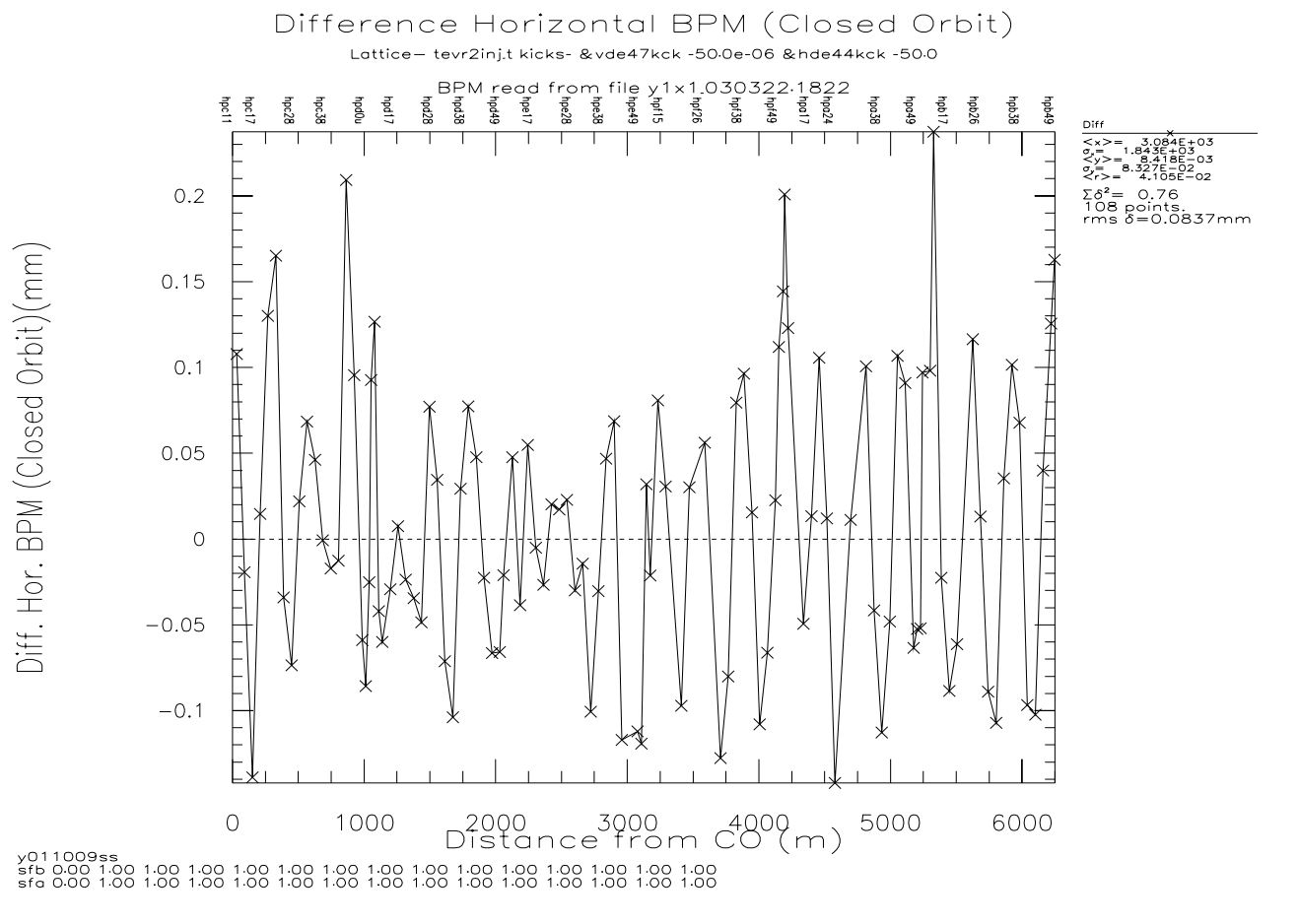

Figure 13 Figure 14 Difference Between the Measured and Calculated Values of the Horizontal BPM Resulting from a -50 $\mu$ r Vertical Kick at E47.

\section{Conclusions}

We have been able to construct a model of the Tevatron, which can reproduce with reasonable accuracy in both transverse planes, the measured dispersion and the closed orbit when a kick is applied in either plane. The crucial element that makes this attempt more successful than earlier attempts in creating a realistic model of the Tevatron is the recent measurements made on coil positions within the dipoles. It has been obvious for some time, at least from the changes in the crossed plane closed orbit, that there were unknown sources of coupling in the Tevatron. These measurements on the magnets provided information on the change in the value of $a_{1}$ from the original MTF measurements. The magnitude of the measured change in $a_{1}$ is what was needed to get a decent fit to the BPM data.

Our model is still not perfect but I think that it represents a significant step forward in our efforts to understand the performance of the Tevatron. Improved BPM would make refinements in the model easier and make the model more accurate.

\section{Acknowledgements.}

I wish to thank Valeri Lebedev for giving me access to the data that he took on the Tevatron. 